\title{
Intranasal administration of cationic liposomes enhanced granulocyte-macrophage colony-stimulating factor expression and this expression is dispensable for mucosal adjuvant activity
}

Rui Tada ${ }^{{ }^{*} \mathbb{D}}$, Akira Hidaka ${ }^{1}$, Hiroshi Kiyono ${ }^{2}$, Jun Kunisawa ${ }^{2,3}$ and Yukihiko Aramaki ${ }^{1}$

\begin{abstract}
Objective: Infectious diseases remain a threat to human life. Vaccination against pathogenic microbes is a primary method of treatment as well as prevention of infectious diseases. Particularly mucosal vaccination is a promising approach to fight against most infectious diseases, because mucosal surfaces are a major point of entry for most pathogens. We recently developed an effective mucosal adjuvant of cationic liposomes composed of 1,2-dioleoyl-

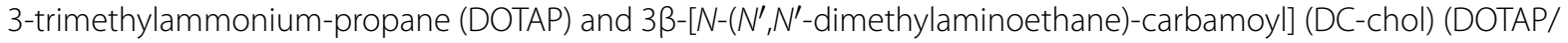
DC-chol liposomes). However, the mechanism(s) underlying the mucosal adjuvant effects exerted by the cationic liposomes have been unclear. In this study, we investigated the role of granulocyte-macrophage colony-stimulating factor (GM-CSF), which was reported to act as a mucosal adjuvant, on the mucosal adjuvant activities of DOTAP/DCchol liposomes when administered intranasally to mice.

Results: Here, we show that, although intranasal vaccination with cationic liposomes in combination with antigenic protein elicited GM-CSF expression at the site of administration, blocking GM-CSF function by using an anti-GM-CSF neutralizing antibody did not alter antigen-specific antibody production induced by DOTAP/DC-chol liposomes, indicating that GM-CSF may not contribute to the mucosal adjuvant activity of the cationic liposomes when administered intranasally.
\end{abstract}

Keywords: Cationic liposome, Granulocyte-macrophage colony-stimulating factor, Intranasal immunization, Mucosal adjuvant

\section{Introduction}

Vaccines have been a great public health success in past decades. However, the development of additional safe and efficient vaccines against various infectious diseases is still a challenge [1-3]. Among vaccine development approaches, mucosal vaccines are most attractive for treating/preventing infectious diseases caused by

\footnotetext{
*Correspondence: rui.tada@gmail.com

${ }^{1}$ Department of Drug Delivery and Molecular Biopharmaceutics, School of Pharmacy, Tokyo University of Pharmacy and Life Sciences, 1432-1, Horinouchi, Hachioji, Tokyo 192-0392, Japan

Full list of author information is available at the end of the article
}

pathogenic microbes, because most pathogens enter the host body via mucosal surfaces $[4,5]$. However, the addition of mucosal adjuvants to mucosal vaccine formulations is crucial to inducing antigen-specific immune responses to proteins derived from microbes, because these antigens show poor immunogenicity in mucosal compartments [6].

We have recently found that nasal immunization of an antigenic protein with cationic liposomes composed of 1,2-dioleoyl-3-trimethylammonium-propane (DOTAP) and $3 \beta-\left[N-\left(N^{\prime}, N^{\prime}\right.\right.$-dimethylaminoethane)carbamoyl] (DC-chol) (DOTAP/DC-chol liposomes) 
potently induced both mucosal and systemic immune responses to the antigen in mice [7-9]. Although the molecular mechanisms underlying the mucosal adjuvant effects exerted by cationic liposomes was unclear, we revealed that these cationic liposomes promote the uptake of antigenic proteins by dendritic cells (DCs) in nasal-associated lymphoid tissues (NALTs) in vivo. In general, one of the molecular mechanisms of adjuvants is increased uptake of antigen and presentation to major histocompatibility complex (MHC) class II on antigen-presenting cells (APCs). However, activation of innate immunity might be much more important for their adjuvant activities, including recruitment of innate immune cells at the site of administration and induction of cytokines and chemokines [10-12]. These views led us to investigate the role of cytokines in the mucosal adjuvant activity of DOTAP/DC-chol liposomes.

In the present study, we hypothesized that granulocyte-macrophage colony-stimulating factor (GM-CSF) might be associated with the mucosal adjuvant effects of DOTAP/DC-chol liposomes administered intranasally to mice. GM-CSF is involved in various biological phenomena, such as promoting cell differentiation, activation, survival, and induction of inflammatory responses [13, 14], as well as inducing mucosal and serum antibody responses when co-administered with antigenic proteins via the nasal route in mice [15-18]. Thus, in this study, we examined the function of GMCSF in DOTAP-DC-chol liposome-induced antigenspecific antibody responses in both mucosal and systemic area in mice.

\section{Main text Methods \\ Mice and materials}

Female BALB/cCrSlc mice (7-10 weeks old) were purchased from Japan SLC (Hamamatsu, Shizuoka, Japan). Animals were housed in a specific pathogen-free environment and all animal experiments were approved by the institution's committee for laboratory animal experiments of the Tokyo University of Pharmacy and Life Sciences (P13-22, P14-31, and P15-33). 1,2-Dioleoyl3 -trimethylammonium-propane (DOTAP) and $3 \beta-[N$ $\left(N^{\prime}, N^{\prime}\right.$-dimethylaminoethane)-carbamoyl $\quad$ (DC-chol) were purchased from Avanti Polar Lipids (Alabaster, AL, USA). Recombinant mouse GM-CSF, anti-GM-CSF neutralizing antibody, and rat IgG2a $\mathrm{K}$ isotype control antibody were all purchased from BioLegend (San Diego, CA, USA). Low endotoxin (less than $1 \mathrm{EU} / \mathrm{mg}$, guaranteed) egg white ovalbumin (OVA) was obtained from Wako Pure Chemical Industries (Osaka, Japan).

\section{Preparation of liposomes}

DOTAP/DC-chol liposomes were prepared as follow [7]. $10 \mu \mathrm{mol}$ of total lipid dissolved in chloroform (DOTAP:DC-chol at a 1:1 mol ratio) was evaporated to dryness to obtain the lipid films. The lipid films were then hydrated in $250 \mu \mathrm{L}$ of phosphate-buffered saline (PBS) and vortexed for $5 \mathrm{~min}$. The prepared liposomes were extruded 10 times by passage through an appropriate pore size polycarbonate membrane (Advantec, Tokyo, Japan) and sterilized via filtration $(0.45-\mu \mathrm{m}$ filter membranes; Iwaki, Tokyo, Japan).

\section{Immunization schedule}

Mice were immunized twice intranasally once a week (days 0 and 7). Mice were divided into three groups as follows: (1) vehicle (PBS), (2) OVA alone (5 $\mu \mathrm{g} /$ mouse), or (3) OVA ( $5 \mu \mathrm{g} / \mathrm{mouse})$ plus liposomes $(400 \mathrm{nmol} / \mathrm{mouse})$ or recombinant mouse GM-CSF $(4 \mu \mathrm{g} /$ mouse described previously [19]). After sacrificing the mice by sodium pentobarbital administration $(100 \mathrm{mg} / \mathrm{kg}$ body weight, intraperitoneal), serum and nasal wash samples were collected on day 14 , as described previously [20,21].

\section{Detection of OVA-specific antibody}

A 96-well plate was coated with OVA in a $0.1 \mathrm{M}$ carbonate buffer ( $\mathrm{pH}$ 9.5). The plate was washed and then blocked with $1 \%$ bovine serum albumin (BSA; Wako Pure Chemical Industries) containing PBST (BPBST) for $60 \mathrm{~min}$ at $37^{\circ} \mathrm{C}$. After washing, the plate was incubated with samples (serum or nasal wash) for $60 \mathrm{~min}$ at $37^{\circ} \mathrm{C}$. To detect OVA-specific IgG antibody, plates were washed with PBST, treated with peroxidase-conjugated anti-mouse IgG (Sigma-Aldrich, St. Louis, MO, USA) in BPBST. The plate was washed, and combined with TMB substrate (KPL, Maryland, USA) and then further incubated for color development. To detect OVA-specific IgA, IgG1, and IgG2a, plates were treated with biotinconjugated anti-mouse IgA, IgG1, or IgG2a (BioLegend) in BPBST, and then avidin-horseradish peroxidase (BioLegend) in PBST was added. Plates were incubated with TMB substrate system (KPL). The reaction was terminated with $1 \mathrm{~N}$ phosphoric acid, and optical densities were measured at $450 \mathrm{~nm} / 650 \mathrm{~nm}$ [22-24]. Endpoint titers were calculated as the reciprocal of the last dilution exceeding a cut-off value that was twice the mean of a negative control $[25,26]$.

\section{Preparation of splenocytes for cell culture}

Splenocytes were prepared as described earlier [13, 27]. Briefly, after sacrificing the mice by cervical dislocation, their spleens were excised and dissociated in RPMI 1640 medium (Wako Pure Chemical Industries). The 
resulting single-cell suspension was then treated with ACK lysis buffer (BioLegend). After centrifugation, splenocytes were suspended in RPMI 1640 medium supplemented with $10 \%$ heat-inactivated fetal bovine serum (FBS; Biowest, Nuaillé, France), $100 \mathrm{U} / \mathrm{mL}$ of penicillin G potassium salt (Sigma-Aldrich), and $100 \mu \mathrm{g} / \mathrm{mL}$ of streptomycin sulfate salt (Sigma-Aldrich). The cells were cultured at $2 \times 10^{6}$ cells/well in $0.5 \mathrm{~mL}$ of culture medium in 48-well flat-bottomed plates (IWAKI) and re-stimulated with OVA (Wako Pure Chemical Industries) for the indicated time at $37^{\circ} \mathrm{C}$ in a $5 \% \mathrm{CO}_{2}$.

\section{Cytokine assay}

The cytokine concentrations were measured using ELISA MAX Standard Sets (BioLegend) according to the manufacturer's instructions. The data were expressed as the mean \pm standard deviation. At least three independent experiments were conducted.

\section{RNA extraction and quantitative real time-polymerase chain reaction ( $q P C R)$}

$\mathrm{BALB} / \mathrm{c}$ female mice were euthanized by sodium pentobarbital administration $(100 \mathrm{mg} / \mathrm{kg}$ body weight, intraperitoneal). Their nasal tissues and spleens were then excised, and the total RNA was extracted from these samples using a FavorPrep Tissue Total RNA Mini Kit (Favorgen Biotech Corporation, Ping-Tung, Taiwan), followed by DNase I (Roche Life Science, Penzberg, Germany) treatment. cDNA was synthesized from total RNA using a ReverTra Ace qPCR RT Master Mix (Toyobo, Tokyo, Japan). Then, qPCR was carried out according to the manufacturer's instructions using a THUNDERBIRD SYBR qPCR Mix (Toyobo). The primers used for PCR were the following: GM-CSF, forward, $5^{\prime}$-TGGGCA TTGTGGTCTACAGC- $3^{\prime}$, and reverse, $5^{\prime}$-GCGGGT CTGCACACATGTTA-3'; $\beta 2$-microglobulin, forward, $5^{\prime}$-TTCTGGTGCTTGTCTCACTGA- $3^{\prime}$, and reverse, 5'-CAGTATGTTCGGCTTCCCATTC-3'. The expression of GM-CSF was determined using the comparative $\Delta$-threshold cycle method using $\beta 2$-microglobulin as a reference gene. GM-CSF expression is presented as the fold change relative to expression in the control sample.

\section{Effect of anti-GM-CSF neutralizing antibody on mucosal adjuvant activity}

$\mathrm{BALB} / \mathrm{c}$ female mice were pre-treated intraperitoneally with anti-GM-CSF neutralizing antibody $(100 \mu \mathrm{g} /$ mouse) 2 days before (day-2) and $1 \mathrm{~h}$ before immunization (days 0 and 7) as reported previously [28-31]. Mice were divided into three groups as follows: (1) PBS, (2) OVA alone ( $5 \mu \mathrm{g} / \mathrm{mouse})$, or (3) OVA ( $5 \mu \mathrm{g} / \mathrm{mouse})$ in combination with liposomes (400 nmol/mouse) on days 0 and 7 . After sacrificing the mice by sodium pentobarbital administration $(100 \mathrm{mg} / \mathrm{kg}$ body weight, intraperitoneal), serum and nasal wash samples were collected on day 14 , as described previously [20,21].

\section{Statistics}

Statistical differences were calculated with unpaired $t$-test with Welch's correction and the Kruskal-Wallis test with Dunn's post hoc test for cytokine and antibody production, respectively. Differences with $p$ values of $<0.05$ were considered significant.

\section{Results \\ Antigen-specific nasal and serum antibodies induced by intranasal immunization of OVA with the cationic liposomes}

First, we evaluated the production of OVA-specific antibodies after intranasal immunization of OVA in combination with DOTAP/DC-chol liposomes in $\mathrm{BALB} / \mathrm{c}$ female mice. As expected, intranasal vaccination induced the production of OVA-specific nasal IgA in nasal fluid and IgG in the serum compartment. In contrast, intranasal immunization with PBS (vehicle) or OVA alone did not exhibit significant OVA-specific antibody production in either mucosal or systemic under these experimental conditions (Additional file 1: Figure S1).

\section{Expression of GM-CSF in mucosal and systemic sites of vaccinated mice}

Prior to assessing the contribution of GM-CSF on the mucosal adjuvant activities of the cationic liposomes, we first examined the expression of GM-CSF at the site of injections. As shown in Fig. 1a, intranasal administration with OVA and DOTAP/DC-chol liposomes significantly exerted the expression of GM-CSF in nasal areas (2.3to 3.3-fold expression compared to mice that received either vehicle or OVA alone). On the other hand, immunization with OVA and DOTAP/DC-chol liposomes did not induce any GM-CSF expression in the spleen. Additionally, since it is known that $\mathrm{T}$ cells activated via TCR signalling are capable of producing a large amount of GM-CSF [32, 33], we investigated antigen-specific GMCSF secretion in splenocytes by re-stimulating them with OVA in vitro. As shown in Fig. 1b, splenocytes from OVA and DOTAP/DC-chol liposome-vaccinated mice produced higher levels of GM-CSF than those from OVA-only administered mice when re-stimulated with OVA. These results indicated that nasally administered DOTAP/DC-chol liposomes were polarized to induce the expression of GM-CSF. 
a GM-CSF expression in nasal tissues
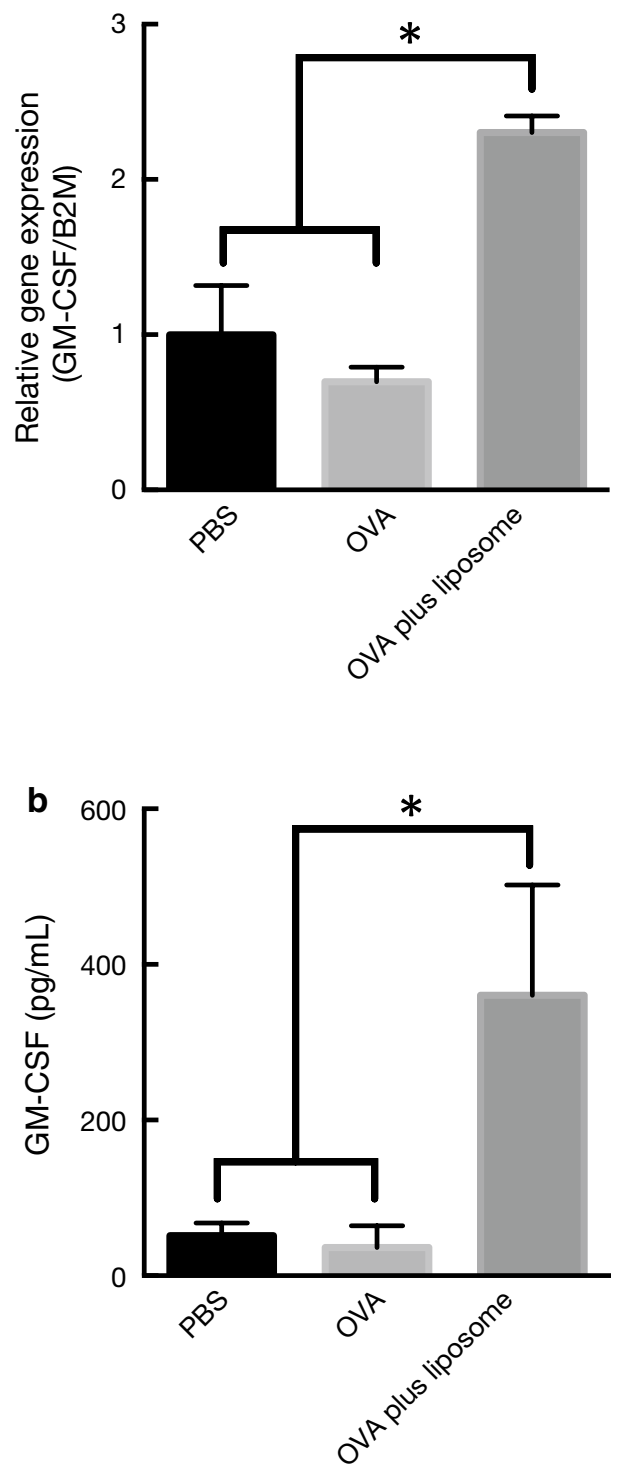

GM-CSF expression in spleen

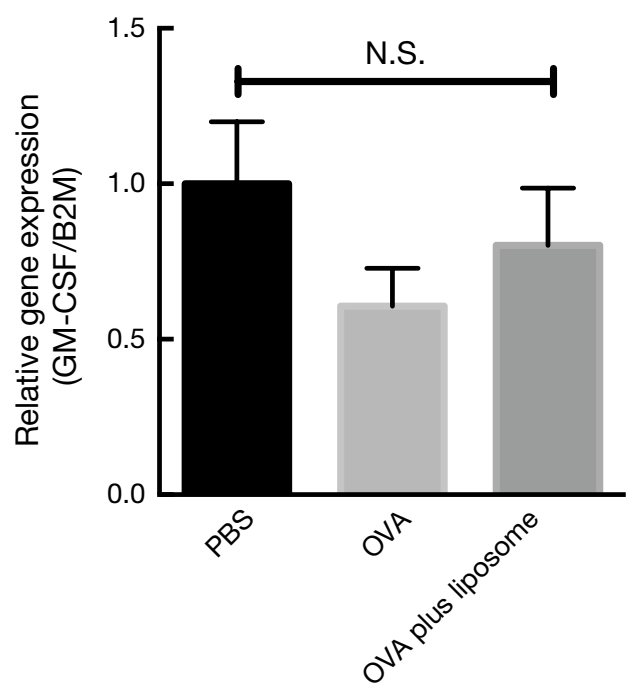

Fig. 1 mRNA expression of granulocyte-macrophage colony-stimulating factor (GM-CSF) in nasal and spleen tissues (a) and antigen-specific production of GM-CSF in splenocytes from mice administered intranasally with OVA plus the liposomes. a Nasal tissue and spleens were collected $6 \mathrm{~h}$ after the last immunization. mRNA expression was measured using QPCR. $\mathbf{b}$ Spleens were collected 1 week after the last immunization, and then harvested splenocytes were cultured for $72 \mathrm{~h}$ in the presence of OVA $(10 \mu \mathrm{g} / \mathrm{mL})$. After culture, concentrations of GM-CSF were determined using ELISA. The values are the mean \pm SD of technical duplicates from three biologically independent experiments. Significance was assessed using unpaired $t$-test with Welch's correction; ${ }^{*} p<0.05$

\section{Effect of GM-CSF on the mucosal adjuvant activities of nasally administered cationic liposomes}

We next explored the role of GM-CSF in the effects of DOTAP/DC-chol liposomes as a mucosal adjuvant when administered intranasally. Before exploring this association, the mucosal adjuvant effect of nasally administered recombinant GM-CSF was examined. As expected, intranasal administration with OVA and recombinant
GM-CSF induced the production of OVA-specific IgA in nasal fluid and IgG in serum samples (Fig. 2), which are almost same that of DOTAP/DC-chol liposomes alone (Additional file 1: Figure S1). Next, we examined whether the induction of mucosal adjuvant effects by DOTAP/ DC-chol liposomes were dependent on GM-CSF using an anti-GM-CSF neutralizing monoclonal antibody (mAb) as previously described to block the biological activities 


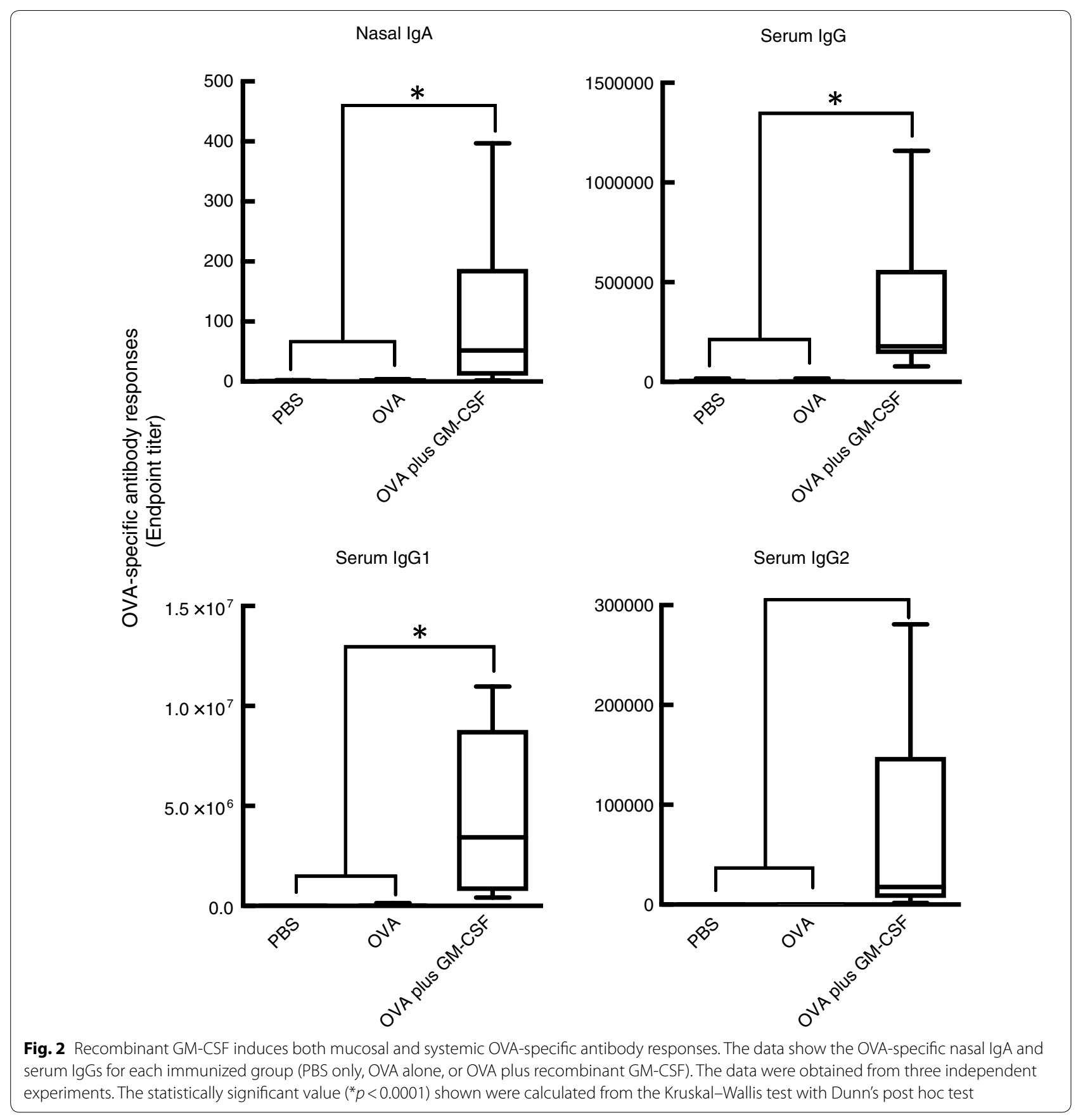

of GM-CSF in vivo [28-31]. Pre-treatment with antiGM-CSF neutralizing antibody did not affect nasal IgA or serum IgG, IgG1, or IgG2 antibody production, showing that GM-CSF expression was not required for the mucosal adjuvant activities of cationic liposomes (Fig. 3).

\section{Discussion}

In this study, we demonstrated the following: (1) intranasal administration with DOTAP/DC-chol liposomes induced the expression of GM-CSF at the site of injections; (2) recombinant GM-CSF showed the mucosal adjuvant effect when nasally administered in mice; and (3) GM-CSF expression in nasal area induced by DOTAP/DC-chol liposomes was not required for the mucosal adjuvant activities.

The development of safe and efficient mucosal adjuvants is needed to prevent fatal infectious diseases. To accomplish this, understanding the mechanism(s) 


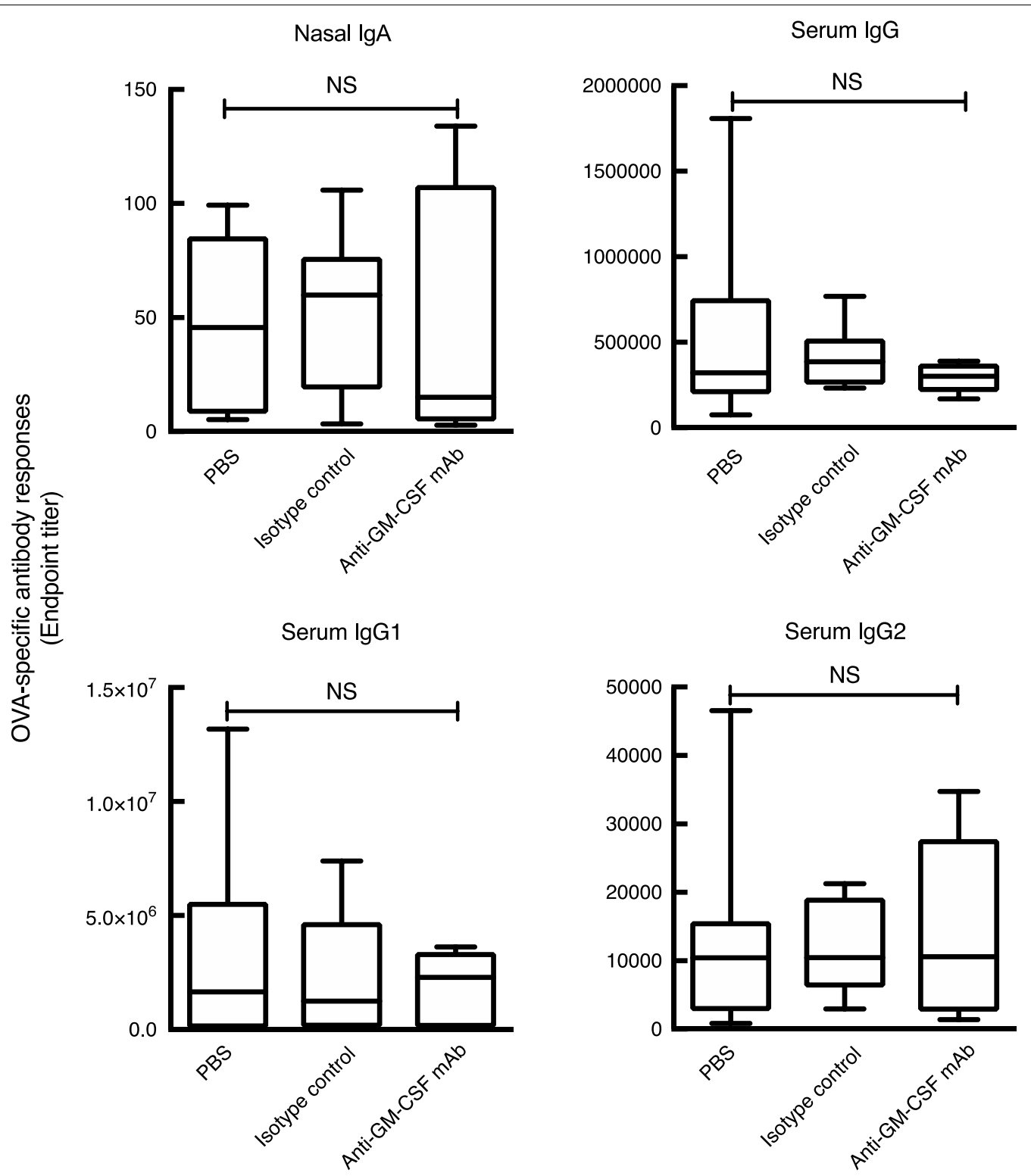

Fig. 3 Effect of anti-GM-CSF neutralizing antibodies on ovalbumin (OVA)-specific antibody responses induced by DOTAP/DC-chol liposomes. Mice were pre-treated intraperitoneally with anti-GM-CSF neutralizing antibody (100 $\mu \mathrm{g} / \mathrm{mouse})$ at day 0 and then immunized intranasally with PBS, OVA alone, or OVA plus DOTAP/DC-chol liposomes on days 2 and 9 . Nasal washes and sera were collected on day 16. OVA-specific nasal IgA and serum IgGs were detected using ELISA. The data were obtained from three independent experiments. NS not significant as evaluated using the KruskalWallis test with Dunn's post hoc test

underlying mucosal adjuvant induction of immune responses to antigenic proteins is essential. Generally, adjuvants show their activities through the depot effect, with the gradual release of antigen at the site of infection and increase in antigen uptake by APCs. Resent research has focused on the role of APCs in activating innate immunity [34]. In particular, the cytokine/chemokine milieu induced by external stimuli, including adjuvants, determines the immune response to antigenic proteins, including the production of antibody to the antigen [3537]. Many studies on the immunomodulating activities of GM-CSF have been reported. For instance, GM-CSF has been shown to stimulate the maturation and function of APCs, such as DCs and macrophages. GM-CSF is also a strong inducer of interleukin-6 (IL-6), which promotes germinal center development and B cell growth and differentiation in these centers [38, 39]. We found that intranasal administration of DOTAP/DC-chol liposomes 
induced IL-6 expression in the nasal mucosa, and that this cytokine was critical for the induction of antigenspecific IgA by the cationic liposomes (unpublished results). Therefore, local GM-CSF expression likely plays a role in enhancing humoral responses to the cationic liposomes. Furthermore, intranasal co-administration of antigens with a GM-CSF-expressing plasmid has been shown to increase OVA-specific mucosal IgA and serum IgG titers, suggesting that GM-CSF plays an essential role in the induction of humoral immune responses to antigenic proteins in both mucosal and systemic compartments $[10,18,19,40]$. We investigated the role of GM-CSF on the mucosal adjuvant activities of the cationic liposomes in this study and found that GM-CSF blocking did not affect their activities, clearly indicating that other soluble factors control the mucosal adjuvant activities of the cationic liposomes. Further experiments are required to clarify the molecular mechanism(s) underlying the induction of humoral immune responses by the cationic liposomes.

\section{Limitations}

Herein, we demonstrated that nasal administration of DOTAP/DC-chol liposomes induced the gene expression of GM-CSF at the site of administration; however, the protein level of GM-CSF in the nasal area after the nasal immunization of DOTAP/DC-chol liposomes has not been evaluated. The major limitation of this study is that though the protocol for studying the biological activities of GM-CSF neutralization at the mucosal compartments by intraperitoneal injection of anti-GM-CSF neutralizing antibody has been reported in literatures [28-31], we have not confirmed these neutralizing effects in this study. Overall, although our data suggested that GM-CSF may not be required for the mucosal adjuvant effects of the cationic liposomes, we were unable to identify the possible mechanism(s) for these effects in this study. Further experiments are required in the future to clarify these aspects.

\section{Additional file}

Additional file 1: Figure S1. DOTAP/DC-chol liposomes potentiate both mucosal and systemic OVA-specific antibody responses. The data show the OVA-specific nasal IgA and serum IgGs for each immunized group (PBS only, OVA alone, or OVA plus liposomes). The data were obtained from three independent experiments. The statistically significant value $\left({ }^{*} p<0.0001\right)$ shown were calculated from the Kruskal-Wallis test with Dunn's post hoc test.

\section{Abbreviations}

APCs: antigen-presenting cells; DC-chol: $3 \beta-\left[N-\left(N^{\prime}, N^{\prime}\right.\right.$-dimethylaminoethane)carbamoyl]; DCs: dendritic cells; DOTAP: 1,2-dioleoyl-3-trimethylammoniumpropane; ELISA: enzyme linked immunosorbent assay; GM-CSF: granulocyte-macrophage colony-stimulating factor; IL-6: interleukin-6; MHC: major histocompatibility complex; NALTs: nasal-associated lymphoid tissues; OVA: ovalbumin from egg white; PBS: phosphate-buffered saline; qPCR: quantitative PCR; TMB: tetramethylbenzidine; TSLP: thymic stromal lymphopoietin.

\section{Authors' contributions}

RT conceived of the study, participated in its design, performed all experiments, analyzed the data and wrote the paper. AH carried out ELISA assay. HK, $J K$ and YA conceived of the study, and participated in its design and coordination and helped to draft the manuscript. All authors read and approved the final manuscript.

\section{Author details \\ ${ }^{1}$ Department of Drug Delivery and Molecular Biopharmaceutics, School of Pharmacy, Tokyo University of Pharmacy and Life Sciences, 1432-1, Horinouchi, Hachioji, Tokyo 192-0392, Japan. ${ }^{2}$ Division of Mucosal Immunol- ogy and International Research and Development Center for Mucosal Vac- cines, Department of Microbiology and Immunology, The Institute of Medi- cal Science, The University of Tokyo, Tokyo, Japan. ${ }^{3}$ Laboratory of Vaccine Materials, National Institutes of Biomedical Innovation, Health and Nutrition (NIBIOHN), Osaka, Japan.}

\section{Acknowledgements}

We are grateful to Saeko Takahashi and Tomoko Iwata for their technical assistance.

\section{Competing interests}

The authors declare that they have no competing interests.

\section{Availability of data and materials}

The datasets used and/or analyzed during the current study available from the corresponding author on reasonable request.

\section{Consent for publication \\ Not applicable.}

\section{Ethics approval and consent to participate}

All animal experiments followed the guidelines for laboratory animal experiments of the Tokyo University of Pharmacy and Life Sciences, and each experimental protocol was approved by the Committee for Laboratory Animal Experiments at the institution (P13-22, P14-31, and P15-33).

\section{Funding}

This study was supported in part by JSPS KAKENHI Grant Number 15K18935 (Grant-in-aid for Young Scientists (B) to RT); JSPS KAKENHI Grant Number 18 K06798 (Grant-in-aid for Scientific Research (C) to RT); JSPS KAKENHI Grant Number 16 K08415 (Grant-in-aid for Scientific Research (C) to YA and RT); $18 \mathrm{H} 02674$ (Grant-in-Aid for Scientific Research (B) to J.K.). The Japan Agency for Medical Research and Development (AMED) (17fk0108223h0002, 17ek0410032s0102, 17fk0108207h0002, 17ak0101068h0001, and

$17 g \mathrm{~m} 101000650101$ to J.K.).

\section{Publisher's Note}

Springer Nature remains neutral with regard to jurisdictional claims in published maps and institutional affiliations.

Received: 15 May 2018 Accepted: 10 July 2018

Published online: 13 July 2018

\section{References}

1. Scully IL, Swanson K, Green L, Jansen KU, Anderson AS. Anti-infective vaccination in the 21st century-new horizons for personal and public health. Curr Opin Microbiol. 2015;27:96-102.

2. Oyston P, Robinson K. The current challenges for vaccine development. J Med Microbiol. 2012;61:889-94.

3. Fauci AS, Touchette NA, Folkers GK. Emerging infectious diseases: a 10-year perspective from the National Institute of Allergy and Infectious Diseases. Emerg Infect Dis. 2005;11:519-25. 
4. Suzuki H, Kondoh M, Yagi K, Kiyono H, Kunisawa J. The development of mucosal vaccine using bacterial function for targeting mucosal tissues. Yakugaku Zasshi. 2014;134:629-34.

5. Kunisawa J, Gohda M, Kiyono H. Uniqueness of the mucosal immune system for the development of prospective mucosal vaccine. Yakugaku Zasshi. 2007;127:319-26.

6. Ada G. Vaccines and vaccination. N Engl J Med. 2001;345:1042-53

7. Tada R, Hidaka A, Iwase N, Takahashi S, Yamakita Y, Iwata T, Muto S, Sato E, Takayama N, Honjo E, et al. Intranasal immunization with DOTAP cationic liposomes combined with DC-cholesterol induces potent antigenspecific mucosal and systemic immune responses in mice. PLoS ONE. 2015;10:e0139785.

8. Tada R, Muto S, Iwata T, Hidaka A, Kiyono H, Kunisawa J, Aramaki Y. Attachment of class B CpG ODN onto DOTAP/DC-chol liposome in nasa vaccine formulations augments antigen-specific immune responses in mice. BMC Res Notes. 2017:10:68.

9. Tada R, Suzuki H, Takahashi S, Negishi Y, Kiyono H, Kunisawa J, Aramaki $Y$. Nasal vaccination with pneumococcal surface protein A in combination with cationic liposomes consisting of DOTAP and DC-chol confers antigen-mediated protective immunity against Streptococcus pneumoniae infections in mice. Int Immunopharmacol. 2018;61:385-93.

10. Thompson AL, Staats HF. Cytokines: the future of intranasal vaccine adjuvants. Clin Dev Immunol. 2011;2011:289597.

11. Boyaka PN, McGhee JR. Cytokines as adjuvants for the induction of mucosal immunity. Adv Drug Deliv Rev. 2001;51:71-9.

12. Boyaka PN, Lillard JW Jr, McGhee J. Interleukin 12 and innate molecules for enhanced mucosal immunity. Immunol Res. 1999;20:207-17.

13. Tada R, Yoshikawa M, Ikeda F, Adachi Y, Kato Y, Kuge T, Tanioka A, Ishibashi K, Tsubaki K, Ohno N. Induction of IFN-gamma by a highly branched 1,3-beta-d-glucan from Aureobasidium pullulans in mouse-derived splenocytes via dectin-1-independent pathways. Biochem Biophys Res Commun. 2011:404:1105-10.

14. Hamilton JA. GM-CSF in inflammation and autoimmunity. Trends Immunol. 2002;23:403-8.

15. Nambiar JK, Ryan AA, Kong CU, Britton WJ, Triccas JA. Modulation of pulmonary DC function by vaccine-encoded GM-CSF enhances protective immunity against Mycobacterium tuberculosis infection. Eur J Immunol. 2010:40:153-61.

16. Ramsburg E, Publicover J, Buonocore L, Poholek A, Robek M, Palin A, Rose JK. A vesicular stomatitis virus recombinant expressing granulocyte-macrophage colony-stimulating factor induces enhanced T-cell responses and is highly attenuated for replication in animals. J Virol. 2005;79:15043-53.

17. Kim HD, Cao Y, Kong FK, Van Kampen KR, Lewis TL, Ma Z, Tang DC, Fukuchi K. Induction of a Th2 immune response by co-administration of recombinant adenovirus vectors encoding amyloid beta-protein and GM-CSF. Vaccine. 2005:23:2977-86.

18. Okada E, Sasaki S, Ishii N, Aoki I, Yasuda T, Nishioka K, Fukushima J, Miyazaki J, Wahren B, Okuda K. Intranasal immunization of a DNA vaccine with IL-12- and granulocyte-macrophage colony-stimulating factor (GM-CSF)-expressing plasmids in liposomes induces strong mucosal and cell-mediated immune responses against HIV-1 antigens. J Immunol. 1997:159:3638-47.

19. Bradney CP, Sempowski GD, Liao HX, Haynes BF, Staats HF. Cytokines as adjuvants for the induction of anti-human immunodeficiency virus peptide immunoglobulin $\mathrm{G}(\mathrm{lgG})$ and $\lg A$ antibodies in serum and mucosal secretions after nasal immunization. J Virol. 2002:76:517-24.

20. Kong IG, Sato A, Yuki Y, Nochi T, Takahashi H, Sawada S, Mejima M, Kurokawa S, Okada K, Sato S, et al. Nanogel-based PspA intranasal vaccine prevents invasive disease and nasal colonization by Streptococcus pneumoniae. Infect Immun. 2013;81:1625-34

21. Suzuki H, Watari A, Hashimoto E, Yonemitsu M, Kiyono H, Yagi K, Kondoh M, Kunisawa J. C-terminal Clostridium perfringens enterotoxinmediated antigen delivery for nasal pneumococcal vaccine. PLoS ONE. 2015;10:e0126352.
22. Tada R, Adachi Y, Ishibashi K, Tsubaki K, Ohno N. Binding capacity of a barley beta-D-glucan to the beta-glucan recognition molecule dectin-1. J Agric Food Chem. 2008;56:1442-50.

23. Tada R, Nagi-Miura N, Adachi Y, Ohno N. Candida albicans derived fungal PAMPS, CAWS, water soluble mannoprotein-beta-glucan complex shows similar immunotoxicological activity with bacterial endotoxin from Escherichia coli O9. Biol Pharm Bull. 2006:29:240-6.

24. Tada R, Tanioka A, Ishibashi K, Adachi Y, Tsubaki K, Ohno N. Involvement of branched units at position 6 in the reactivity of a unique variety of beta-D-glucan from Aureobasidium pullulans to antibodies in human sera. Biosci Biotechnol Biochem. 2009;73:908-11.

25. Gillgrass AE, Ashkar AA, Rosenthal KL, Kaushic C. Prolonged exposure to progesterone prevents induction of protective mucosal responses folowing intravaginal immunization with attenuated herpes simplex virus type 2. J Virol. 2003;77:9845-51.

26. Lu G, Gonzalez R, Guo L, Wu C, Wu J, Vernet G, Paranhos-Baccala G, Wang J, Hung T. Large-scale seroprevalence analysis of human metapneumovirus and human respiratory syncytial virus infections in Beijing, China. Virol J. 2011;8:62

27. Tada R, Yoshikawa M, Kuge T, Tanioka A, Ishibashi K, Adachi Y, Tsubaki K, Ohno N. A highly branched 1,3-beta-D-glucan extracted from Aureobasidium pullulans induces cytokine production in DBA/2 mouse-derived splenocytes. Int Immunopharmacol. 2009;9:1431-6.

28. Willart MA, Deswarte K, Pouliot P, Braun H, Beyaert R, Lambrecht BN, Hammad H. Interleukin-1alpha controls allergic sensitization to inhaled house dust mite via the epithelial release of GM-CSF and IL-33. J Exp Med. 2012;209:1505-17.

29. Khajah M, Millen B, Cara DC, Waterhouse C, McCafferty DM. Granulocyte-macrophage colony-stimulating factor (GM-CSF): a chemoattractive agent for murine leukocytes in vivo. J Leukoc Biol. 2011;89:945-53.

30. Vlahos R, Bozinovski S, Chan SP, Ivanov S, Linden A, Hamilton JA, Anderson GP. Neutralizing granulocyte/macrophage colony-stimulating factor inhibits cigarette smoke-induced lung inflammation. Am J Respir Crit Care Med. 2010;182:34-40.

31. Laan M, Prause O, Miyamoto M, Sjostrand M, Hytonen AM, Kaneko T, Lotvall J, Linden A. A role of GM-CSF in the accumulation of neutrophils in the airways caused by IL-17 and TNF-alpha. Eur Respir J. 2003;21:387-93.

32. Griffin JD, Cannistra SA, Sullivan R, Demetri GD, Ernst TJ, Kanakura Y The biology of GM-CSF: regulation of production and interaction with its receptor. Int J Cell Cloning. 1990;8(Suppl 1):35-44 (discussion 44-35).

33. Shi Y, Liu CH, Roberts Al, Das J, Xu G, Ren G, Zhang Y, Zhang L, Yuan $\mathrm{ZR}$, Tan HS, et al. Granulocyte-macrophage colony-stimulating factor (GM-CSF) and T-cell responses: what we do and don't know. Cell Res. 2006:16:126-33.

34. Awate S, Babiuk LA, Mutwiri G. Mechanisms of action of adjuvants. Front Immunol. 2013:4:114

35. Zhu J, Yamane H, Paul WE. Differentiation of effector CD4 T cell populations (*). Annu Rev Immunol. 2010;28:445-89.

36. Huse M, Lillemeier BF, Kuhns MS, Chen DS, Davis MM. T cells use two directionally distinct pathways for cytokine secretion. Nat Immunol. 2006;7:247-55.

37. Spellberg B, Edwards JE Jr. Type 1/type 2 immunity in infectious diseases. Clin Infect Dis. 2001:32:76-102.

38. Evans R, Shultz LD, Dranoff G, Fuller JA, Kamdar SJ. CSF-1 regulation of 116 gene expression by murine macrophages: a pivotal role for GM-CSF. J Leukoc Biol. 1998:64:810-6.

39. Burdin N, Galibert L, Garrone P, Durand I, Banchereau J, Rousset F. Inability to produce IL-6 is a functional feature of human germinal center B lymphocytes. J Immunol. 1996;156:4107-13.

40. Loudon PT, Yager EJ, Lynch DT, Narendran A, Stagnar C, Franchini AM, Fuller JT, White PA, Nyuandi J, Wiley CA, et al. GM-CSF increases mucosal and systemic immunogenicity of an $\mathrm{H} 1 \mathrm{~N} 1$ influenza DNA vaccine administered into the epidermis of non-human primates. PLOS ONE. 2010;5:e11021 